\title{
Erlotinib as standard adjuvant therapy for resectable EGFR mutation-positive non-small cell lung cancer
}

\author{
Satomi Tanaka, Junji Uchino \\ Department of Pulmonary Medicine, Kyoto Prefectural University of Medicine, Kyoto, Japan \\ Correspondence to: Dr. Junji Uchino. Department of Pulmonary Medicine, Kyoto Prefectural University of Medicine, 465 Kajii-cho, Hirokoji-agaru, \\ kawaramachi-dori, Kamigyo-ku, Kyoto, Kyoto 602-0857 Japan. Email: uchino@koto.kpu-m.ac.jp. \\ Provenance: This is an invited article commissioned by the Section Editor Hengrui Liang (Department of Thoracic Surgery, Guangzhou Medical \\ University, Guangzhou, China). \\ Comment on: Yue D, Xu S, Wang Q, et al. Erlotinib versus vinorelbine plus cisplatin as adjuvant therapy in Chinese patients with stage IIIA EGFR \\ mutation-positive non-small-cell lung cancer (EVAN): a randomised, open-label, phase 2 trial. Lancet Respir Med 2018;6:863-73.
}

Submitted May 11, 2019. Accepted for publication Jun 11, 2019.

doi: $10.21037 /$ tlcr.2019.06.05

View this article at: http://dx.doi.org/10.21037/tlcr.2019.06.05

This Chinese randomized, open-label, phase 2 trial compared erlotinib with the standard-of-care vinorelbine plus cisplatin therapy as adjuvant treatment for patients with stage IIIA EGFR mutation-positive non-small cell lung cancer (NSCLC) who were able to undergo complete resection.

A total of 102 patients with stage IIIA EGFR mutationpositive NSCLC that was completely resectable were enrolled from 16 Chinese centers and assigned to receive erlotinib (i.e., the erlotinib group) or vinorelbine plus cisplatin therapy (i.e., the chemotherapy group), and their 2 -year disease-free survival was evaluated. The 2 -year disease-free survival rate was $81.4 \%$ in the erlotinib group and $44.6 \%$ in the chemotherapy group. The difference between the 2 groups was significant at $36.7 \%(\mathrm{P}=0.0007)$.

Adverse events were observed in $29 / 50$ patients (58\%) in the erlotinib group and $28 / 43$ patients $(65 \%)$ in the chemotherapy group. The most common adverse events were rash in the erlotinib group (2/50 patients, $4 \%$ ) and neutropenia (7/43 patients, $16 \%$ ) and myelosuppression (4/43 patients, $9 \%)$ in the chemotherapy group, with no treatment-related deaths reported. Thus, erlotinib was more effective and tolerable than vinorelbine plus cisplatin when used as adjuvant treatment.

Cytocidal anticancer regimens are the current standardof-care for postoperative adjuvant chemotherapy for patients with completely resectable stage IIIA NSCLC. A metaanalysis based on individual data from 4,584 patients, i.e., the Lung Adjuvant Cisplatin Evaluation (LACE), showed significant survival benefit with adjuvant chemotherapy, with a hazard ratio (HR) of 0.83 (95\% CI: 0.72-0.94) for stage III cancer patients (1). On subgroup analyses, the survival outcome after cisplatin plus vinorelbine therapy had a HR of 0.80 (95\% CI: $0.70-0.91$ ), with a significant survival benefit. In addition, a meta-analysis of 34 known studies with 8,447 cases showed good clinical results $(2,3)$.

Although epidermal growth factor receptor-tyrosine kinase receptor (EGFR-TKI) is the standard-of-care treatment for advanced-stage EGFR mutation-positive NSCLC, the efficacy of EGFR-TKI has not been established for resectable EGFR mutation-positive NSCLC. Several trials have compared cytocidal anticancer agents with EGFR-TKI for patients with completely resectable NSCLC, but none are positive studies, and EGFR-TKI is currently not recommended as postoperative adjuvant chemotherapy for stage IIIA NSCLC that is completely resectable (4-6). However, these studies used EGFR-TKI for cases with or without EGFR mutations. Moreover, a small percentage of EGFR mutation-positive patients have been studied in EGFR mutation-positive groups only through subset analyses. For example, in the BR19 trial, Caucasians accounted for $90 \%$ of all enrollees, with $15 / 359$ $E G F R$-positive cases and approximately $13 \%$ of stage IIIA cases (5). The RADIANT trial was also conducted for determining the efficacy of erlotinib (6). Subgroup analyses limited to the EGFR mutation-positive group did not show prolonged survival (the median survival was not reached in both erlotinib- and placebo-treated patients; HR, 1.09; 95\% 
CI: $0.55-2.16)$. The current study also included Caucasian patients, accounting for $80 \%$ of the total population; moreover, 161/973 patients were positive for EGFR mutations, and approximately $15 \%$ of the total patients had stage IIIA disease (6).

As noted above, other occasional reports have described that the frequency of EGFR mutation-positive lung cancer, response to TKI treatment, and incidence of adverse events differ according to race $(7,8)$. Regarding treatment efficacy for advanced-stage lung cancer, a subgroup analysis of a phase III trial evaluating the efficacy of second-line erlotinib therapy showed a response rate of $18.9 \%$ in Asians and $7.5 \%$ in patients of other races (8). Moreover, in phase III trials evaluating sequential treatment with afatinib and osimertinib, the median duration of treatment was 46.7 months (95\% CI: 26.8-not reached) in Asians versus 27.6 months (95\% CI: 24.5-29.2) in non-Asians (9). In contrast, in the osimertinib FLAURA trial, the overall response rate was $80 \%$ (95\% CI: $75-85 \%$ ) in the osimertinib group and $76 \%$ (95\% CI: $70-81 \%$ ) in the standard EGFR-TKI group, compared with $80 \%$ (95\% CI: $73-86 \%)$ in the osimertinib group and $75 \%$ (95\% CI: 68-82\%) in the standard EGFR-TKI group on the Asian subset analysis, suggesting differences in effects between races depending on the type of TKI (10). This study was limited to Chinese subjects, and it is very interesting to note that the efficacy of adjuvant treatment with EGFR-TKIs in Asians complements the abovementioned data.

In the 2-year disease-free survival assessment, patients in the chemotherapy arm completed 4 cycles of cisplatin plus vinorelbine whereas those in the erlotinib arm received oral medication for 2 years. In addition, the optimal duration of erlotinib treatment was unclear because no evaluation has been performed after completion of oral erlotinib treatment. In addition, future research on the administration period is needed considering the cost and quality of life associated with erlotinib administration for 2 years.

Among adverse events, the occurrence of interstitial pneumonia should also be monitored. Severe interstitial lung disease (ILD) is common in Asians, particularly Japanese people, and the incidence of Grade 3-4 ILD is $2 \%$ in Japanese patients treated with gefitinib and $0 \%$ in non-Japanese patients. Erlotinib also caused ILD in $4.6 \%$ of Japanese patients in phase II studies (11-13). In this study, severe ILD was reported in 1 of the 50 patients in the erlotinib group; although the incidence was not very different from that reported previously, the overall number of patients in the erlotinib group was small and thus, the safety assessment seems inadequate.

The small sample size should be listed as a limitation. The reason for setting the HR at 0.5 is ambiguous, and the fact that the $\alpha$ error was set as 0.2 also leaves questions about the certainty of the test results. Furthermore, the chemotherapy group protocol-compliant population included only 33 patients (58\% of the intent to treat population), and the validity of such a control group is questionable.

Hence, further studies are needed to establish erlotinib as an adjuvant treatment for patients with stage IIIA cancer.

Thus, the EVAN trial has demonstrated the efficacy of EGFR-TKI as an adjuvant treatment for EGFR-positive NSCLC. In addition, similar reports have been sporadically published since 2018, when the EVAN trials have been published. Two studies were conducted on gefitinib in China in 2018: the ADJUVANT/CTONG 1104 trials and a retrospective study $(14,15)$. The former compared gefitinib with cisplatin plus vinorelbine therapy whereas the latter compared gefitinib with platinum doublet therapy. Both studies have shown good DFS in the gefitinib group. The WJOG6410L trials are currently evaluating gefitinib for stage II-III EGFR-positive NSCLC after complete resection, and the results are awaited. The SELECT trials, conducted in the United States in 2018, evaluated erlotinib for stage IA-IIIA NSCLC after resection and showed a 2 -year DFS of $85 \%$ (16). Osimertinib is also undergoing the ADAURA trial (NCT02511106) as an adjuvant treatment for stage IB-IIIA NSCLC. The results of this study are also expected to provide outcome- and safety-related data of osimertinib against advanced-stage lung cancer.

As of 2014, there have been reports of randomized phase 2 trials of pemetrexed + carboplatin \pm gefitinib as adjuvant chemotherapy for $E G F R$-positive stage IIIA-N2 NSCLC after complete resection in Chinese patients (17). The gefitinib-combination group also showed significantly improved DFS [HR 0.37 (95\% CI: 0.16-0.85)]. Postoperative adjuvant chemotherapy with platinum doublet and EGFR-TKI may be an option for stage IIIA $E G F R$-positive NSCLC in the future.

The duration of EGFR-TKI administration in adjuvant therapy remains an issue. The current EVAN trial has a 2-year duration, and the ADAURA trial has a duration of up to 3 years. Gefitinib was also administered for 2 years in the BR19 trial (4) and in the ADJUVANT/CTONG 1104 trial, which evaluated the abovementioned EGFRTKI as the first adjuvant therapy. The WJOG6410L trials (results awaited) also involves gefitinib administration for 
2 years. The trial of pemetrexed + carboplatin \pm gefitinib included chemotherapy followed by 6 months of gefitinib administration. Erlotinib was also administered for 2 years in the RADIANT and SELECT trials, with the majority of patients receiving erlotinib for 2 years. The FLAURA trial involving patients with advanced-stage NSCLC reported a progression-free survival of 18.9 months (95\% CI: 15.2 21.4), and no evidence is available supporting the safety of using the same TKIs for at least 2 years. Therefore, 2 years is considered to be an appropriate administration period for TKIs at present, but the results of the ADAURA trial, evaluating internal use for 3 years, are awaited.

Treatment of unresectable stage IIIB-IV NSCLC is undergoing dramatic transformation owing to the development in EGFR-TKI and immune-checkpoint inhibitors. Moreover, the development of microarrays and next-generation sequencing is expected to result in personalized medicine for the treatment of advanced-stage lung cancer according to the nature of the tumor in the future.

Currently, there is a critical paucity of data regarding postoperative adjuvant treatment after curative resection of stage IIIA NSCLC, but the accumulation of trial results, such as those of this study, is expected to introduce personalized medicine for postoperative adjuvant treatment of resectable NSCLC. Thus, the accumulation of studies examining the effects and tolerability of drugs in limited populations, such as this study, will provide important data in this era of personalized medicine.

\section{Acknowledgments}

None.

\section{Footnote}

Conflicts of Interest: The authors have no conflicts of interest to declare.

Ethical Statement: The authors are accountable for all aspects of the work in ensuring that questions related to the accuracy or integrity of any part of the work are appropriately investigated and resolved.

\section{References}

1. Pignon JP, Tribodet H, Scagliotti GV, et al. Lung adjuvant cisplatin evaluation: a pooled analysis by the LACE
Collaborative Group. J Clin Oncol 2008;26:3552-9.

2. Douillard JY, Tribodet H, Aubert D, et al. Adjuvant cisplatin and vinorelbine for completely resected non-small cell lung cancer: subgroup analysis of the Lung Adjuvant Cisplatin Evaluation. J Thorac Oncol 2010;5:220-8.

3. Arriagada R, Auperin A, Burdett S, et al; NSCLC Metaanalyses Collaborative Group. Adjuvant chemotherapy, with or without postoperative radiotherapy, in operable non-small-cell lung cancer: two meta-analyses of individual patient data. Lancet 2010;375:1267-77.

4. Tsuboi M, Kato H, Nagai K, et al. Gefitinib in the adjuvant setting: safety results from a phase III study in patients with completely resected non-small cell lung cancer. Anticancer Drugs 2005;16:1123-8.

5. Goss GD, O'Callaghan C, Lorimer I, et al. Gefitinib versus placebo in completely resected non-small-cell lung cancer: results of the NCIC CTG BR19 study. J Clin Oncol 2013;31:3320-6.

6. Kelly K, Altorki NK, Eberhardt WE, et al. Adjuvant Erlotinib Versus Placebo in Patients With Stage IBIIIA Non-Small-Cell Lung Cancer (RADIANT): A Randomized, Double-Blind, Phase III Trial. J Clin Oncol 2015;33:4007-14.

7. Sekine I, Yamamoto N, Nishio K, et al. Emerging ethnic differences in lung cancer therapy. Br J Cancer 2008;99:1757-62.

8. Shepherd FA, Rodrigues Pereira J, Ciuleanu T, et al. Erlotinib in previously treated non-small-cell lung cancer. N Engl J Med 2005;353:123-32.

9. Hochmair MJ, Morabito A, Hao D, et al. Sequential treatment with afatinib and osimertinib in patients with EGFR mutation-positive non-small-cell lung cancer: an observational study. Future Oncol 2018;14:2861-74.

10. Soria JC, Ohe Y, Vansteenkiste J, et al. Osimertinib in Untreated EGFR-Mutated Advanced Non-Small-Cell Lung Cancer. N Engl J Med 2018;378:113-25

11. Fukuoka M, Yano S, Giaccone G, et al. Multi-institutional randomized phase II trial of gefitinib for previously treated patients with advanced non-small-cell lung cancer (The IDEAL 1 Trial). J Clin Oncol 2003;21:2237-46.

12. Kris MG, Natale RB, Herbst RS, et al. Efficacy of gefitinib, an inhibitor of the epidermal growth factor receptor tyrosine kinase, in symptomatic patients with non-small cell lung cancer: a randomized trial. JAMA 2003;290:2149-58.

13. Yamamoto N, Horiike A, Fujisaka Y, et al. Phase I dosefinding and pharmacokinetic study of the oral epidermal growth factor receptor tyrosine kinase inhibitor Ro50- 
8231 (erlotinib) in Japanese patients with solid tumors. Cancer Chemother Pharmacol 2008;61:489-96.

14. Zhong WZ, Wang Q, Mao WM, et al. Gefitinib versus vinorelbine plus cisplatin as adjuvant treatment for stage II-IIIA (N1-N2) EGFR-mutant NSCLC (ADJUVANT/ CTONG1104): a randomised, open-label, phase 3 study. Lancet Oncol 2018;19:139-48.

15. Xie H, Wang H, Xu L, et al. Gefitinib Versus Adjuvant Chemotherapy in Patients With Stage II-IIIA Non-SmallCell Lung Cancer Harboring Positive EGFR Mutations: A Single-Center Retrospective Study. Clin Lung Cancer

Cite this article as: Tanaka S, Uchino J. Erlotinib as standard adjuvant therapy for resectable EGFR mutation-positive nonsmall cell lung cancer. Transl Lung Cancer Res 2019;8(Suppl 4):S369-S372. doi: 10.21037/tlcr.2019.06.05
2018;19:484-92.

16. Pennell NA, Neal JW, Chaft JE, et al. SELECT: A Phase II Trial of Adjuvant Erlotinib in Patients With Resected Epidermal Growth Factor Receptor-Mutant Non-SmallCell Lung Cancer. J Clin Oncol 2019;37:97-104.

17. Li N, Ou W, Ye X, et al. Pemetrexed-carboplatin adjuvant chemotherapy with or without gefitinib in resected stage IIIA-N2 non-small cell lung cancer harbouring EGFR mutations: a randomized, phase II study. Ann Surg Oncol 2014;21:2091-6. 